\title{
MAXIMALLY ALGEBRAIC POTENTIALLY IRRATIONAL CUBIC FOURFOLDS
}

\author{
RADU LAZA
}

\begin{abstract}
A well known conjecture asserts that a cubic fourfold $X$ whose transcendental cohomology $T_{X}$ can not be realized as the transcendental cohomology of a $K 3$ surface is irrational. Since the geometry of cubic fourfolds is intricately related to the existence of algebraic 2-cycles on them, it is natural to ask for the most algebraic cubic fourfolds $X$ to which this conjecture is still applicable. In this paper, we show that for an appropriate "algebraicity index" $\kappa_{X} \in \mathbb{Q}_{+}$, there exists a unique class of cubics maximizing $\kappa_{X}$, not having an associated $K 3$ surface; namely, the cubic fourfolds with an Eckardt point (previously investigated in [LPZ17]). Arguably, they are the most algebraic potentially irrational cubic fourfolds, and thus a good testing ground for the Harris, Hassett, Kuznetsov conjectures.
\end{abstract}

\section{INTRODUCTION}

A celebrated result of Clemens and Griffiths CG72 says that a smooth cubic threefold $Y$ is irrational. This follows by showing that while the Hodge structure on $H^{3}(Y)$ looks like (the Tate twist of) the Hodge structure of a curve, it is in fact not coming (over $\mathbb{Z}$ ) from a curve. Similarly, for cubic fourfolds, one sees that the Hodge structure on $H^{4}(X)_{\text {prim }}$ is of $K 3$ type. In analogy with the Clemens-Griffiths result, it is natural to conjecture that if the transcendental cohomology of $X$ is not actually coming from a $K 3$ surface $S$ (i.e. $H^{4}(X)_{\mathrm{tr}} \cong H^{2}(S)(-1)_{\mathrm{tr}}$ ), then $X$ is not rational. This was proposed by Harris, and some evidence was given by Hassett Has96. Has99: all known rational cubic fourfolds have transcendental cohomology (over $\mathbb{Z}$ ) induced from a $K 3$ surface (see also [AT14 for a discussion of the related Kuznetsov conjecture). However, we emphasize that no example of irrational cubic fourfold (or for that matter any irrational cubic of dimension > 3) is currently known. There are numerous papers on the subject of cubics and rationality, but the focus so far seems to have been on (Hodge) general cubics or almost general cubics (e.g. cubics containing a plane). In this paper, we turn the question around and ask:

Question 1.1. Assuming that there exist irrational cubic fourfolds, which are the maximally algebraic cubic fourfolds $X$ for which the rationality fails?

For a cubic $X$, its algebraicity is naturally measured by the rank of the group of primitive algebraic cycles

$$
\rho_{X}:=\operatorname{rank}\left(H^{2,2}(X) \cap H^{4}(X, \mathbb{Z})_{\text {prim }}\right) \in\{0, \ldots, 20\} .
$$

For a general cubic fourfold $X, \rho_{X}=0$. In general, $\rho_{X}=k$ is a codimension $k$ condition on moduli. More precisely, due to the theory of periods for cubic fourfolds (Voi86], Has00, [Laz09b, Laz10, Loo09]), for $k \geq 1$, the locus of cubics with $\rho_{X} \geq k$ is a countable union of codimension $k$ closed algebraic subvarieties in the moduli space $\mathcal{M}$ of cubic fourfolds. These special loci can be indexed (compare [Has00]) by a second positive integer

$$
d_{X}:=\operatorname{det}\left(H^{2,2}(X) \cap H^{4}(X, \mathbb{Z})\right),
$$

(N.B. the associated locus might be empty or reducible for some values of the pair $\left.\left(\rho_{X}, d_{X}\right)\right)$. It is natural to understand maximally algebraic cubic as both maximizing $\rho_{X}$ and minimizing $d_{X}$. We propose to combine $\left(\rho_{X}, d_{X}\right)$ in a single value algebraicity index for $X$, defined by

$$
\kappa_{X}=\frac{2^{\rho_{X}}}{d_{X}} \in \mathbb{Q}_{+} .
$$

Thus, a more precise version of Question (1.1) is to ask to maximize $\kappa_{X}$ subject to the irrationality constraint.

Date: May 11, 2018.

Research of the author is supported in part by NSF grants DMS-1254812 and DMS-1361143. 
Remark 1.5. Vinberg Vin83 studied the two most algebraic $K 3$ surfaces. Similar to the $K 3$ case, the two most algebraic cubic fourfolds (i.e. those giving maximal values for $\kappa_{X}$ ) are the (rigid) cubics with transcendental lattices: $A_{2}(-1)=\left(\begin{array}{cc}-2 & 1 \\ 1 & -2\end{array}\right)$ and $\left(A_{1}(-1)\right)^{2}=\left(\begin{array}{cc}-2 & 0 \\ 0 & -2\end{array}\right)$ respectively (same, up to a twist, as for $K 3 \mathrm{~s})$. For these two examples, we get $\kappa_{X}=\frac{2^{21}}{3}$ and $2^{19}$ respectively. In contrast, as we will discuss below, the cubics that are conjectured to be irrational have $\kappa_{X} \leq 1$.

No example of irrational cubic fourfold $X$ is known; we have nothing new to say in this direction. Thus, for all we know, it is possible that Question (1.1) is void. Nonetheless, we use it as motivation to answering the following more tangible question:

Question 1.6. Which cubic fourfolds $X$, without associated $K 3$ surfaces (in the sense of [Has96]), maximize the algebraicity index $\kappa_{X}$ ?

As discussed, the Harris-Hassett conjecture predicts that a cubic $X$ without an associated $K 3$ surface is irrational. Thus, the two questions are related modulo a hard open conjecture. Without touching on this conjecture, the goal of our paper is to bring about a different perspective on it. Namely, on a general cubic fourfold, it is difficult to do geometry as there are no special algebraic cycles to build on. As we will see below, our answer $X$ to Question 1.6 is at the opposite end: e.g. $X$ contains 27 planes. This is surprising, as a cubic fourfold $X$ containing two disjoint planes not only has an associated $K 3$, but it is in fact rational.

Definition 1.7. Let $X$ be a smooth cubic fourfold. Let $N_{X}:=H^{2,2}(X) \cap H^{4}(X, \mathbb{Z})$ be the lattice of algebraic cycles, and $T_{X}=\left(N_{X}\right)_{H^{4}(X, \mathbb{Z})}^{\perp}$ be the transcendental lattice. We say that $X$ is potentially irrational if the transcendental lattice $T_{X}$ does not admit a primitive embedding into the (Tate twisted) $K 3$ lattice $\left(E_{8}\right)^{2} \oplus U^{3}$. (In the literature, potentially irrational means $X$ does not have an associated $K 3$ surface.)

Our main result is a full answer to Question 1.6.

Theorem 1.8. Let $X$ be a cubic fourfold.

(0) If $X$ is potentially irrational, then $\kappa_{X} \leq 1$.

(1) A general cubic fourfold $X$ containing an Eckardt point is potentially irrational with $\kappa_{X}=1$.

(2) Conversely, any potentially irrational $X$ with $\kappa_{X}=1$ is a cubic fourfold with an Eckardt point.

The cubics occurring in the theorem above were investigated in our paper [LPZ17] with G. Pearlstein and Z. Zhang. They arise in connection with our search for pseudo-cubics (i.e. Fano fourfolds with middle cohomology of $K 3$ type). As reviewed in Section 4, there are multiple geometric characterization of such cubics. The most relevant fact here is that they admit an Eckardt point $p \in X$. This implies that $X$ contains 27 planes passing through $p$. It then follows that the lattice of primitive algebraic cycles is isometric to $E_{6}(2)$. In [LPZ17] we studied the moduli space of cubic fourfolds with an Eckardt point by viewing them as $E_{6}(2)$-marked cubics (in analogy to the $M$-polarized $K 3$ s of Dolgachev).

Theorem 1.8 (see 2.11 4.3 and 3.8 for proofs of items (1), (2), and (3) respectively) follows from the geometric considerations of [LPZ17, and Nikulin's theory of lattices ([Nik79b, Nik79a]). We have been inspired by the papers of Vinberg Vin83 and D. Morrison Mor84 which touch on similar questions for $K 3$ s. An expert look at the lattices involved in our paper (i.e. $E_{6}(2)$ and $\left.\left(D_{4}\right)^{3} \oplus U^{2}\right)$ will bring into focus another special features of the cubics $X$ studied in [LPZ17. Namely, their Fano variety $F(X)$ of lines admits an exotic anti-symplectic involution, i.e. an involution which is not induced (via deformations) from a $K 3$ surface. In contrast, for symplectic involutions on hyper-Kähler fourfolds of $K 3^{[2]}$ type, Mongardi Mon12] proved that they are all induced from $K 3$ surfaces (N.B. this was generalized recently to the $K 3^{[n]}$ type by Kamenova and Mongardi). For higher orders, Mongardi Mon13 and others produced examples of exotic symplectic automorphisms on hyper-Kähler fourfolds of $K 3^{[2]}$ type (via constructions involving cubics).

Convention 1.9. The root lattices $A D E$ are positive definite. For a lattice $L, L(a)$ denotes the lattice scaled by $a$. Depending on the context, the " $K 3$ lattice" refers either to $\left(E_{8}(-1)\right)^{2} \oplus U^{3}$ or $\left(E_{8}\right)^{2} \oplus U^{3}$.

Acknowledgement. We have been aware of the [LPZ17 cubics since the author's thesis in connection to the deformations of the $O_{16}$ singularity (the cone over a cubic surface). We thank R. Friedman and I. Dolgachev for many stimulating discussions on the subject. Many of the ideas in this paper crystallized as consequence of discussions with G. Pearlstein and Z. Zhang around our joint work [LPZ17. Finally, this paper was prompted by a brief chance discussion with L. Kamenova and A. Kumar on a related topic. 


\section{General Considerations on potentially irrational Cubic fourfolds}

As a consequence of Torelli theorems, Question 1.6 is equivalent to the following lattice theoretic question.

Question 2.1. Let $T$ be an even lattice of signature $(n, 2)$ admitting a primitive embedding into the lattice $A_{2} \oplus\left(E_{8}\right)^{2} \oplus U^{2}$. When does $T$ admit a primitive embedding into the K3 lattice $E_{8}^{2} \oplus U^{3}$ ?

Remark 2.2. A similar question was asked by D. Morrison [Mor84: Which K3 surfaces are Kummer? Equivalently, Let $T$ be a sublattice of the K3 lattice, when does $T$ embed into the Kummer lattice $U^{3}$ ?

We fix the following assumptions and notations consistent with the Introduction (except for dropping the index $X$, as the discussion here is a purely lattice theoretic).

Notation 2.3. Let $T$ be an even lattice of signature $(n, 2)$ with $n \leq 20$. Assume given a fixed primitive embedding

$$
T \hookrightarrow I_{21,2}
$$

such that $N:=T^{\perp}$ contains a element $h$ with $h^{2}=3$ and such that $\langle h\rangle_{I_{21,2}}^{\perp}$ is an even lattice (we say $h$ is of even type). The choice of $h$ is unique up to the action of $O\left(I_{21,2}\right)$, and assumed fixed throughout. An easy application of [Nik79b] shows that $\Lambda_{0}:=\langle h\rangle_{I_{21,2}}^{\perp} \cong A_{2} \oplus\left(E_{8}\right)^{2} \oplus U^{2}$. We let

$$
N_{0}:=\langle h\rangle_{N}^{\perp} .
$$

The lattices $T$ and $N_{0}$ are mutually orthogonal lattices in $\Lambda_{0}$. Frequently, it is more convenient to consider $T$ as a sublattice of $\Lambda_{0}$, and define $N_{0}$ as its orthogonal complement with respect to this embedding. The extension to an embedding $T$ into $I_{21,2}$ (and similarly of $N_{0}$ to $N$ ) is unique. As before, we let

$$
\begin{aligned}
\rho & :=\operatorname{rank} N_{0}(=22-n), \\
d & :=\operatorname{det}(T) .
\end{aligned}
$$

(and $\kappa=\frac{2 \rho}{d}$ ). For a lattice $L$, we denote by $A_{L}=L^{*} / L$ the discriminant group. If $L$ is an even lattice, $A_{L}$ comes endowed with a finite quadratic form $q_{L}: A_{L} \rightarrow \mathbb{Q} / 2 \mathbb{Z}$. The key invariant relevant in the discussion below is the discriminant group $A_{T} \cong A_{N}$ and the number of its invariant factors:

$$
\ell=l\left(A_{T}\right) .
$$

Nikulin's theory Nik79b provides a complete answer to embedding questions such as those raised in Question 2.1. A key aspect of Nikulin's results is that, in most cases, deciding that a lattice $L$ admits an embedding into some other lattice (typically unimodular) reduces to comparing two integers. Specifically, in our situation, most of the cases of Question 2.1 can be decided by comparing the invariants $\ell$ and $\rho$.

Proposition 2.5. With notation as above.

i) A necessary condition that $T$ embeds into the $K 3$ lattice is $\ell \leq \rho$;

ii) Conversely, if $\ell \leq \rho-1$, there exists a primitive embedding of $T$ into the $K 3$ lattice. Furthermore, if $\ell \leq \rho-2$, the embedding is unique.

Proof. Assume $T$ embeds into the $K 3$ lattice with orthogonal complement $M$. Then, $A_{T} \cong A_{M}$. It follows

$$
\ell=l\left(A_{T}\right)=l\left(A_{M}\right) \leq \min (\operatorname{rank} T, \operatorname{rank} M)=\min (\rho, 22-\rho) .
$$

The converse follows from [Nik79b, Cor. 1.12.3].

Remark 2.6. The assumption $T$ embeds into the lattice $I_{21,2}$, automatically gives

$$
\ell \leq \min (\rho+1,22-\rho) .
$$

If $\ell=\rho+1$, then $T$ does not admit an embedding into the $K 3$ lattice (cf. Prop. 2.5(1)). Thus, the associated cubic fourfolds $X$ (with $T=T_{X}$ ) are potentially irrational in the sense of Def. 1.7. An example is given by $X$ a general cubic fourfold, for which we have $T \cong A_{2} \oplus E_{8}^{2} \oplus U^{2}, \rho=0$, and $\ell=1$. Note that the algebraicity index is $\kappa_{X}=\frac{1}{3}$ for a general cubic fourfold (cf. (1.4)) 
Remark 2.8. Continuing the previous remark, let us note that for most cases $\ell \leq \rho$, and that if this fails (i.e. $\ell=\rho+1)$, then the algebraicity index $\kappa$ is small. First, note that $N_{0}$ and $T$ are mutually orthogonal in $\Lambda_{0}$ and $A_{\Lambda_{0}} \cong \mathbb{Z} / 3$. It follows that either

$$
A_{T} \equiv A_{N_{0}} / \mathbb{Z} / 3
$$

or $A_{T}$ is a $\mathbb{Z} / 3$ extension of $A_{N_{0}}$. In the first case, $\ell \leq \rho$. While in the second case, the only possibility that $\ell=\rho+1$ is for the 3 -part of $A_{T}$ to have $\rho+1$ generators. Thus

$$
3^{\rho+1}|\operatorname{det} T=| A_{T} \mid \text {. }
$$

which in turn gives $\kappa \leq \frac{1}{3}$; the equality holds only for general cubic fourfolds.

In conclusion, the only interesting case for us is $\ell=\rho$. Furthermore, from Prop. 2.5 and (2.7), we note that if $\rho$ is big, then $T$ embeds into the $K 3$ lattice.

Proposition 2.9. If $\rho \geq 11$, then $T$ admits a primitive embedding into the $K 3$ lattice.

Proof. $T$ has signature $(20-\rho, 2)$. Note that $20-\rho \leq 9$, and the proposition is precisely Mor84, Cor. 2.10 and Rem. 2.11] (based on [Nik79b, Thm. 1.10.1]).

In conclusion, the maximal $\rho_{X}$ that can occur on a potentially irrational cubic fourfold $X$ is 10 (N.B. we do not know if $\rho_{X}=10$ is achieved for some $\left.X\right)$. We expect a full classification of the cases $\left(\rho_{X}, d_{X}\right)$ for which $X$ is potentially irrational to be delicate as it involves the difficult case $(\ell=\rho)$ of Nikulin's theory. For instance, we recall the following result of Hassett which answers the case $\rho=1$. We are also aware of some partial results for $\rho=2$ (e.g. [AT14, [ABBVA14).

Theorem 2.10 (Hassett Has00, Thm. 1.0.2]). Let $X$ be a cubic fourfold and $d \equiv 0,2$ (mod 6$)$. Assume $\rho_{X}=1$ and $\operatorname{det} T_{X}=d$. Then $X$ is potentially irrational iff one of the following holds:

a) $d \equiv 0(\bmod 9)$

b) $d \equiv 0(\bmod 4)$

c) $d$ has an odd prime factor $p \equiv-1(\bmod 3)$.

Our algebraicity index $\kappa=\frac{2^{\rho}}{d}$ is controlling the growth of the rank $\rho$ relative to (log of) the size $d$ of the discriminant group (i.e. $\log _{2} \kappa=\rho-\log _{2} d$ ). It turns out that it is easy to classify the cases maximizing $\kappa$. As a first step, we establish item (1) of Theorem 1.8 .

Corollary 2.11. If $X$ is a potentially irrational cubic fourfold. Then $\kappa_{X} \leq 1$. Furthermore, if the equality holds, then $T_{X}$ is a 2-elementary lattice.

Proof. Let $T:=T_{X}$ be the transcendental cohomology of $X$. By assumption $T$ does not admit a primitive embedding into the $K 3$ lattice. By Proposition 2.5 and Remark 2.6 $\ell \in\{\rho, \rho+1\}$. Since $\ell$ is the minimal number of generators of the abelian group $A_{T}$, and $d$ is the order of $A_{T}$, we conclude

$$
d \geq 2^{\ell} \geq 2^{\rho} .
$$

The equality holds iff $A_{T} \cong(\mathbb{Z} / 2)^{\rho}$, which is to say $T$ is 2-elementary.

\section{The 2-Elementary CASE}

The 2-elementary lattices have been recognized by Nikulin Nik79a as playing a key role in the geometry of $K 3$ surfaces; for instance they control the possible involutions on a $K 3$ surface.

3.1. Nikulin's classification of 2-elementary lattices. Let $M$ be a 2-elementary lattice, i.e. $A_{M} \cong$ $(\mathbb{Z} / 2)^{l}$. In addition to the obvious invariants of $M$ : signature $\left(t_{+}, t_{-}\right)$and $l=l\left(A_{M}\right)$, there is a parity invariant $\delta$ defined by

$$
\delta:=\left\{\begin{array}{lc}
0 & \text { if } q_{M} \text { takes values in } \mathbb{Z} / 2 \mathbb{Z} \subset \mathbb{Q} / 2 \mathbb{Z} \\
1 & \text { else. }
\end{array}\right.
$$

Example 3.2. $D_{4}$ and $U(2)$ are 2-elementary lattices with $\delta=0$. While $A_{1}$ and $E_{7}$ have $\delta=1$.

Theorem 3.3 (Nikulin, see [Dol83, Thm. 1.5.2]). The genus of an even 2-elementary lattice $M$ is determined by the invariants $\delta, l$ and $\left(t_{+}, t_{-}\right)$. If $M$ is indefinite, then the genus consists of one isomorphism class. An even 2-elementary lattice $M$ with invariants $\delta, l$, and $\left(t_{+}, t_{-}\right)$exists iff the following conditions are satisfied: 
0) $l, t_{+}, t_{-} \geq 0, \delta \in\{0,1\}$;

1) $t_{+}+t_{-} \geq l$;

2) $t_{+}+t_{-}+l \equiv 0(\bmod 2)$;

3) if $\delta=0$, then $t_{+}-t_{-} \equiv 0(\bmod 4)\left(\right.$ e.g. $\left.U(2), D_{4}\right)$;

4) if $l=0$, then $\delta=0, t_{+}-t_{-} \equiv 0(\bmod 8)$ (e.g. unimodular);

5) if $l=1$, then $\left|t_{+}-t_{-}\right| \equiv 1(\bmod 8)\left(\right.$ e.g. $\left.A_{1}, E_{7}\right)$;

6) if $l=2$ and $t_{+}-t_{-} \equiv 4(\bmod 8)$, then $\delta=0\left(\right.$ e.g. $\left.U(2), D_{4}\right)$;

7) if $\delta=0$ and $l=t_{+}+t_{-}$, then $t_{+}-t_{-} \equiv 0(\bmod 8)\left(\right.$ e.g. $\left.U(2), E_{8}(2)\right)$.

3.2. Potentially irrational cubics with 2-elementary transcendental lattice. We are returning to our set-up (in particular, notations as in 2.3). In the context of Cor. 2.11, we assume that $T$ is a 2-elementary lattice and that $T$ does admit a primitive embedding into the $K 3$ lattice.

Theorem 3.4. The only 2-elementary lattice $T$ of signature $(20-\rho, 2)$ that embeds into $A_{2} \oplus\left(E_{8}\right)^{2} \oplus U^{2}$, but does not embed into the $K 3$ lattice $\left(E_{8}\right)^{2} \oplus U^{3}$ is $T=\left(D_{4}\right)^{3} \oplus U^{2}$ (with $\operatorname{sign}=(14,2), l=6$, and $\left.\delta=0\right)$.

Proof. By Propositions 2.5 and 2.9] we can restrict to the case $\ell=\rho \leq 10$ (here $l=\ell$ ). By Nikulin [Nik79b, Thm. 1.12.2] $T$ admits an embedding into the $K 3$ lattice iff there exists a 2-elementary lattice $M$ (recall $\left.A_{M} \cong A_{T}\right)$ with invariants sign $=(1, \rho-1), l=\rho$, and $\delta=\delta_{T}$. Inspecting Theorem 3.3, we see that if $\delta=1$, there is no further restriction on the existence of $M$ (or, more elementary, we can take $M=A_{1} \oplus\left(A_{1}(-1)\right)^{\rho}$, corresponding geometrically to a double cover of $\mathbb{P}^{2}$ branched in a sextic with $\rho(\leq 10)$ nodes). Thus, $T$ as in the theorem should have additionally $\delta_{T}=0$. Under this assumption, the condition (3) of Theorem 3.3 gives $\rho \equiv 2(\bmod 4)$. There are 3 values in the range of interest here: $\rho \in\{2,6,10\}$. The condition $(7)$ of 3.3 then says that $M$ exists iff $\rho \equiv 2(\bmod 8)$ (for the existence part, we can take $U(2)$ (i.e. hyperelliptic $K 3$ ) and $U(2) \oplus E_{8}(2)$ (i.e. double cover of Enriques) respectively; the subtle part, where we need Thm. 3.3, is the non-existence of $M$ for $\rho=6$ ).

We conclude that the only potential case for $T$ is the 2-elementary lattice with invariants sign $=(14,2)$, $l=6$, and $\delta=0$. Since $T$ is indefinite, the isometry class of $T$ is determined by the invariants. Since $\left(D_{4}\right)^{3} \oplus U^{2}$ and $T$ have the same invariants, we conclude $T \cong\left(D_{4}\right)^{3} \oplus U^{2}$. The existence of an embedding of $T$ into the cubic lattice was established in [LPZ17. In particular, we note that with respect to the embedding $T \hookrightarrow A_{2} \oplus\left(E_{8}\right)^{2} \oplus U^{2}$ considered in LPZ17, the orthogonal complement of $T$ is $E_{6}(2)$.

To complete the proof of item (3) of Theorem 1.8, we need to conclude that the embedding of $T$ into $A_{2} \oplus\left(E_{8}\right)^{2} \oplus U^{2}$ is unique. By Nikulin's theory, this boils down to the statement that $E_{6}(2)$ is unique in its genus. This is probably well known, but for lack of a reference, we provide a proof.

Lemma 3.5. The only isometry class in the genus of $E_{6}(2)$ is $E_{6}(2)$.

Proof. Assume $L$ is a lattice in the genus of $E_{6}(2)$. Thus, $L$ is even positive definite and

$$
A_{L} \cong A_{E_{6}(2)} \cong \mathbb{Z} / 3 \times(\mathbb{Z} / 2)^{6} .
$$

Furthermore, the finite quadratic form $q_{L}[2]$ on $A_{L}[2]\left(:=3 A_{L}\right)$ takes only integral values (i.e. it maps to $\mathbb{Z} / 2 \mathbb{Z} \subset \mathbb{Q} / 2 \mathbb{Z})$.

Claim 3.6. The norm of any vector $L$ (a lattice in the genus of $E_{6}(2)$ is divisible by 4 .

Proof (Claim). If not, let $v \in L$ with $v^{2} \equiv 2(\bmod 4)$. Let $L^{\prime}:=\langle v\rangle_{L}^{\perp}$. We can view $L$ as an overlattice of $\langle v\rangle \oplus L^{\prime}$. Following [Nik79b, §1.4], there exists a gluing group $H$ with $H \subset A_{\langle v\rangle} \oplus A_{L^{\prime}}$ such that

$$
A_{L} \cong H^{\perp} / H
$$

where the orthogonal complement is taken in $A_{\langle v\rangle} \oplus A_{L^{\prime}}$ with respect to the finite quadratic form $q_{\langle v\rangle} \oplus q_{L^{\prime}}$ (and $H$ is totally isotropic w.r.t. to $q_{\langle v\rangle} \oplus q_{L^{\prime}}$; in particular, $H \subset H^{\perp}$ ). Let us focus on the 2-Sylow subgroups of the various discriminant groups. First, since $v \equiv 2(\bmod 4)$, we get $A_{\langle v\rangle}[2] \cong \mathbb{Z} / 2$. On the other hand, $l\left(A_{L^{\prime}}[2]\right) \leq 5$ and $l\left(A_{L}[2]\right)=6$. We conclude that $H[2]$ is trivial. Thus, we have a direct sum decomposition

$$
A_{L}[2] \cong A_{\langle v\rangle}[2] \oplus A_{L^{\prime}}[2] .
$$

This clearly contradicts (since $A_{\langle v\rangle}[2]$ forces $\delta=1$ ) the fact that $A_{L}[2]$ takes only values in $\mathbb{Z} / 2 \mathbb{Z} \subset \mathbb{Q} / 2 \mathbb{Z}$. 
Returning to the lemma, as a consequence of the claim, we also have $v \cdot w \equiv 0(\bmod 2)$ for any $v, w \in L$ (e.g. $\left.2\langle v, w\rangle=(v+w)^{2}-v^{2}-w^{2}\right)$. We conclude that we can rescale $L$ by $\frac{1}{2}$. Thus $L(1 / 2) \cong E_{6}=E_{6}(2 \cdot 1 / 2)$ (as $E_{6}$ is unique in its genus). The claim follows.

Theorem 3.7. Let $T=\left(D_{4}\right)^{3} \oplus U^{2}$. Then there exists a unique primitive embedding of $T$ into the lattice $\Lambda_{0}=A_{2} \oplus\left(E_{8}\right)^{2} \oplus U^{2}$ (up to $O^{*}\left(\Lambda_{0}\right)$ ). This extends to a unique embedding $T$ into the cubic lattice $I_{21,2}$.

Proof. The embedding $T \hookrightarrow \Lambda_{0}$ depends on the isometry class of $M=T_{\Lambda_{0}}^{\perp}$ and the choice of gluing subgroup $H \subset A_{T} \oplus A_{M}$ (with $\left.H^{\perp} / H \cong A_{\Lambda_{0}} \cong \mathbb{Z} / 3\right)$. Since $A_{T} \cong(\mathbb{Z} / 2)^{6}, A_{\Lambda_{0}} \cong \mathbb{Z} / 3$, and $M$ is positive definite of rank 6, we conclude $A_{M} \cong A_{A_{2}} \oplus A_{T}$ and $M \cong H$. Since $E_{6}(2)$ and $T$ admit mutually orthogonal embeddings in $\Lambda_{0}$, we conclude that $M$ is in the same genus as $E_{6}(2)$ (as there is a unique choice of invariants). By Lemma 3.5. $M \cong E_{6}(2)$ (and thus there is no choice for $M$ ).

It remains to consider the choice of gluing group $H$. As noted, the natural projection $H \subset A_{M} \oplus A_{T} \rightarrow A_{T}$ induces an isomorphism $H \cong A_{T}\left(\cong(\mathbb{Z} / 2)^{6}\right)$. Fixing an identification of the 2-primary part in $A_{M}$ with $A_{T}$, we see that the choices of $H$ induce automorphisms of $A_{T}(\cong \mathbb{Z} / 2)^{6}$, and in fact they are in 1-to-1 correspondence with the isometries in $O\left(q_{T}\right) \cong W\left(E_{6}\right)$. Finally, since $T$ contains two hyperbolic summands $U$, the natural morphism $O(T) \rightarrow O\left(q_{T}\right)$ is surjective ([Nik79b, Thm. 1.14.2]), concluding the proof of the fact that $T$ admits a unique embedding into $\Lambda_{0}$ modulo $O^{+}\left(\Lambda_{0}\right)$. Since $A_{\Lambda_{0}} \cong \mathbb{Z} / 3$ is identified with the 3 -primary part of $A_{M}$, and since the gluing group $H$ only involves the 2-primary part, all choices involved (and identifications made) respect the discriminant $A_{\Lambda_{0}}$. Thus, we get uniqueness modulo $O^{*}\left(\Lambda_{0}\right)$.

The lattice $\Lambda$ is obtained by glueing $\Lambda_{0}$ and $\langle h\rangle$ (with $h^{2}=3$ ) along $H \cong \mathbb{Z} / 3$. Arguing as above, we can chose the gluing vector $v$ (i.e. $v$ such that $v+h$ is divisible by 3 in $\Lambda$ ) in $M$. The claim follows.

At this point, we conclude that there is only one class of maximally algebraic potentially irrational cubics.

Corollary 3.8. The Zariski closure of the locus of potentially irrational cubic fourfolds $X$ with $\kappa_{X}=1$ is an irreducible 14 dimensional algebraic subvariety in the moduli space $\mathcal{M}$ of cubic fourfolds

Proof. By Corollary 2.11 and Theorem 3.4, we conclude that the transcendental lattice $T_{X}$ of a cubic fourfold as in the statement of the corollary is isometric to $\left(D_{4}\right)^{3} \oplus U^{2}$. By Theorem 3.7 $T_{X}$ admits a unique embedding into the primitive cubic lattice $\Lambda_{0}$. Thus, we can uniquely (up to monodromy) mark a transcendental lattice $T \cong\left(D_{4}\right)^{3} \oplus U^{2}$ inside $H^{4}(X, \mathbb{Z})$. It follows that the locus of cubics with periods belonging to $T$ is an irreducible subvariety $Z$ (of codimension 6 ) of the period domain $\mathcal{D} / \Gamma$.

Recall $\mathcal{D} / \Gamma$ is a quasi-projective variety (Baily-Borel Theorem), and that there exists a period map

$$
\mathcal{P}: \mathcal{M} \rightarrow \mathcal{D} / \Gamma \backslash\left(\mathcal{C}_{2} \cup \mathcal{C}_{6}\right),
$$

that is an isomorphism of quasi-projective varieties (cf. [Voi86, Laz10, Loo09). We conclude that $\mathcal{P}^{-1}\left(Z \backslash\left(\mathcal{C}_{2} \cup \mathcal{C}_{6}\right)\right) \subset \mathcal{M}$ is an irreducible subvariety in the moduli space $\mathcal{M}$ of cubic fourfolds parameterizing (the closure of the locus of) maximally algebraic potentially irrational cubic fourfolds.

A final point here is that the divisors $\mathcal{C}_{2}$ and $\mathcal{C}_{6}$ that are missing from the image of the period map (cf. [Has00, Laz10, Loo09]) have (from a lattice theoretic point of view) associated $K 3$ surfaces, of degree 2 and 6 respectively. This implies that the locus $Z$ will not be completely contained in $\mathcal{C}_{2} \cup \mathcal{C}_{6}$. Thus $\mathcal{P}^{-1}\left(Z \backslash\left(\mathcal{C}_{2} \cup \mathcal{C}_{6}\right)\right) \neq \emptyset$ and of expected dimension.

Remark 3.9. As always with cubic fourfolds (esp. those containing planes), the (potential) irrationality rest on a subtle index 2 issue. Specifically, if instead of $T_{X}=\left(D_{4}\right)^{3} \oplus U^{2} \cong D_{4} \oplus E_{8} \oplus U(2) \oplus U(2)$ we are considering the index 2 overlattice $D_{4} \oplus E_{8} \oplus U(2) \oplus U$, then there exists an associated $K 3$ surface $S$ with Neron-Severi lattice $M=D_{4} \oplus U(2)$. A geometric realization for $S$ can be obtained via the double cover of $\mathbb{P}^{2}$ branched in a quintic and a line. There is a close geometric relationship between $X$ (with $T_{X}$ as above) and $S$. Both cases arise from the study of certain singularities $\left(O_{16}\right.$ and $N_{16}$ respectively; see [Laz09a]).

\section{Cubic Fourfolds with an Eckardt point}

The only remaining issue is to provide a geometric meaning for cubic fourfolds $X$ with transcendental cohomology $T_{X} \cong\left(D_{4}\right)^{3} \oplus U^{2}$. The content of [LPZ17] is to analyze the moduli space of cubics $X$ with an Eckardt point $p$, which turn out to have transcendental lattice precisely $\left(D_{4}\right)^{3} \oplus U^{2}$. In view of Corollary 3.8, this concludes the proof of Theorem 1.8 
The cubic fourfolds considered in LPZ17 arise from multiple different perspectives, and have many special properties. Let us give a brief overview of the situation. First, motivated by the study of the singularity $O_{16}$ (see Laz09a]), we can consider cubic pairs $(Y, S)$ consisting of a cubic threefold and a hyperplane section $S$ of $Y$. By a construction analogous to [ACT11, we can encode such a pair $(Y, S)$ into a cubic fourfold $X$ together with an involution $\iota$ fixing a hyperplane. Explicitly, we can choose coordinates on $\mathbb{P}^{5}$ such that

$$
X=V\left(f_{3}\left(x_{0}, \ldots, x_{4}\right)+x_{0} x_{5}^{2}\right)
$$

where $f_{3}$ is a general cubic form (with $Y=V\left(f_{3}\right) \subset \mathbb{P}^{4}$, and $S=V\left(f_{3}, x_{0}\right)$ ). The cubic $X$ is characterized by the fact that it carries an involution $\iota$ (explicitly $x_{5} \rightarrow-x_{5}$ ) fixing a hyperplane (here $V\left(x_{5}\right)$ ) and a point $p$ (here $p=(0, \ldots, 0,1))$. It turns out that $p$ is an Eckardt point for $X$ (i.e. $X \cap T_{p} X$ is the cone over the cubic surface $S$ ), and that this can be used to give a different geometric characterization of $X$. Finally, $X$ also occurs as a smooth birational model for a general degree 6 weighted hypersurface in $W \mathbb{P}(1,2,2,2,2,3)$ (which occurs in the analysis of the four-dimensional analogue of Reid's list of $95 K 3$ surfaces that are weighted hypersurfaces; this was our original motivation for [LPZ17]). All these geometric characterizations are shown to be equivalent in [LPZ17. The relevant bit here is the following:

Theorem 4.2 ([LPZ17]). For a general cubic fourfold $X$ as in [LPZ17, the following hold:

i) $X$ contains 27 planes $\Pi_{i}$ passing through the Eckardt point p;

ii) The primitive algebraic cohomology $N_{0} \cong E_{6}(2)$ (spanned by classes $\left[\Pi_{i}\right]-\left[\Pi_{j}\right]$ );

iii) The transcendental cohomology of $X$ is $T \cong\left(D_{4}\right)^{3} \oplus U^{2}$.

Corollary 4.3. A general cubic fourfold $X$ containing an Eckardt point is maximally algebraic potentially irrational. Conversely, any maximally algebraic potentially irrational $X$ is a cubic with a single Eckardt point.

\section{REFERENCES}

[ABBVA14] A. Auel, M. Bernardara, M. Bolognesi, and A. Várilly-Alvarado, Cubic fourfolds containing a plane and a quintic del Pezzo surface, Algebr. Geom. 1 (2014), no. 2, 181-193.

[ACT11] D. Allcock, J. A. Carlson, and D. Toledo, The moduli space of cubic threefolds as a ball quotient, Mem. Amer. Math. Soc. 209 (2011), no. 985, xii+70.

[AT14] N. Addington and R. Thomas, Hodge theory and derived categories of cubic fourfolds, Duke Math. J. 163 (2014), no. $10,1885-1927$.

[CG72] C. H. Clemens and P. A. Griffiths, The intermediate Jacobian of the cubic threefold, Ann. of Math. (2) 95 (1972), 281-356.

[Dol83] I. V. Dolgachev, Integral quadratic forms: applications to algebraic geometry (after V. Nikulin), Bourbaki seminar, Vol. 1982/83, Astérisque, vol. 105, Soc. Math. France, Paris, 1983, pp. 251-278.

[Has96] B. Hassett, Special cubic hypersurfaces of dimension four, Ph.D. thesis, Harvard University, 1996.

[Has99] Some rational cubic fourfolds, J. Algebraic Geom. 8 (1999), no. 1, 103-114.

[Has00] S S Secial cubic fourfolds, Compositio Math. 120 (2000), no. 1, 1-23.

[Laz09a] R. Laza, Deformations of singularities and variation of GIT quotients, Trans. Amer. Math. Soc. 361 (2009), no. 4, 2109-2161.

[Laz09b] ㄴ. The moduli space of cubic fourfolds, J. Algebraic Geom. 18 (2009), no. 3, 511-545.

[Laz10] The moduli space of cubic fourfolds via the period map, Ann. of Math. (2) 172 (2010), no. 1, 673-711.

[Loo09] E. Looijenga, The period map for cubic fourfolds, Invent. Math. 177 (2009), no. 1, 213-233.

[LPZ17] R. Laza, G. Pearlstein, and Z. Zhang, On the moduli space of pairs consisting of a cubic threefold and a hyperplane, arXiv:1710.08056, 2017.

[Mon12] G. Mongardi, Symplectic involutions on deformations of K3 ${ }^{[2]}$, Cent. Eur. J. Math. 10 (2012), no. 4, $1472-1485$.

[Mon13] _ Automorphisms of Hyperkähler manifolds, Ph.D. thesis, Università degli Studi di Roma Tre, 2013, arXiv:1303.4670.

[Mor84] D. R. Morrison, On K3 surfaces with large Picard number, Invent. Math. 75 (1984), no. 1, $105-121$.

[Nik79a] V. V. Nikulin, Finite groups of automorphisms of Kählerian K3 surfaces, Trudy Moskov. Mat. Obshch. 38 (1979), $75-137$.

[Nik79b] Integer symmetric bilinear forms and some of their geometric applications, Izv. Akad. Nauk SSSR Ser. Mat. 43 (1979), no. 1, 111-177, 238.

[Vin83] È. B. Vinberg, The two most algebraic K3 surfaces, Math. Ann. 265 (1983), no. 1, 1-21.

[Voi86] C. Voisin, Théorème de Torelli pour les cubiques de $\mathbf{P}^{5}$, Invent. Math. 86 (1986), no. 3, 577-601.

Stony Brook University, Stony Brook, NY 11794, USA

E-mail address: radu.laza@stonybrook.edu 